\title{
Stakeholders' perspectives on improving adolescents' dietary behaviours in the school setting: a qualitative study
}

\author{
L.D. Devine ${ }^{1}$, A.M. Gallagher ${ }^{1}$ and A.J. Hill ${ }^{1}$ \\ ${ }^{1}$ Nutrition Innovation Centre for Food and Health, School of Biomedical Sciences, Ulster University, Coleraine, UK
}

This abstract was awarded the student prize.

The transitional period from childhood to adolescence is recognised as a nutritionally vulnerable time as adolescents begin to develop their own sense of autonomy and thus their propensity towards food choice becomes more independent-based ${ }^{(1)}$. Schools are favourable environments to intervene and positively influence food-related behaviours during adolescence as eating habits are less resistant to change than in later life ${ }^{(2)}$. This study aimed to explore school stakeholders' perspectives on adolescents' food choices within the post-primary school setting and identify feasible strategies that could promote selecting healthier options in this population.

A purposive sample of 29 school stakeholders in post-primary schools and the Education Authority (EA) were recruited from a range of geographical locations across Northern Ireland (NI). Preliminary analysis has been completed for 16 stakeholders (teachers $(\mathrm{n}=8)$; principal/ vice principal $(\mathrm{n}=2)$; catering staff $(\mathrm{n}=5)$ ) within 10 post-primary schools in urban and rural areas and a senior staff member $(n=1)$ within the EA. School stakeholders were invited to participate in one-to-one remote interviews which were undertaken via telephone or Microsoft Teams between October 2020 and December 2020. Written consent was provided prior to commencing the interviews. Stakeholders' views on the school food environment, current school practices, factors influencing adolescents' dietary choices in the school canteen and suggestions on how to promote improved eating habits in this age group were elicited using a semi-structured discussion guide. Interviews were on average 30 minutes and were audio-recorded, transcribed verbatim and analysed thematically following an inductive approach on NVivo12 software ${ }^{(3)}$. This study was approved by Ulster University's Research Ethics Committee.

Results of the preliminary analysis $(n=16)$ identified seven themes for factors influencing adolescents' food choices in the school canteen, namely: peer influence; family influence (parental influence, home eating habits); environmental-related factors (convenience, assortment, food availability, queueing, time restrictions); nutritional knowledge (non-influential in food selection); financial motivations (price, value for money); personal preference (taste, appearance, habitual intake) and age group (junior students versus senior students dietary habits). Strategies to encourage healthier school-based food behaviours could be considered under eight themes, namely: incentives (financial, social, recognition and educational rewards); student-led initiatives; school staff involvement; advertisement and promotion of healthier options; food-related improvements (food quality, food offerings); environmental-related improvements (assortment, menu availability); meal deals and educational approaches (educational talks, educational resources).

Analysis is ongoing and the results will be updated to include 29 stakeholders. Following this, the next stage is to interview adolescents in year 8 and 9 on their food choices within the school setting and their recommendations on how best to improve eating behaviours. These findings will inform the design of a school-based dietary intervention suitable for this population which will be delivered and evaluated in NI post-primary schools in 2021/22.

\section{Acknowledgements}

The authors thank the teachers, vice principals, principals, caterers and Education Authority staff who participated in this study. This study was undertaken as part of a PhD scholarship funded by the Department for the Economy (DfE).

\section{References}

1. McEvoy CT, Lawton J, Kee F, et al. (2014) Health Educ Res 29, 799-811

2. Calvert S, Dempsey RC \& Povey R (2019) Obes Rev 20, 543-553

3. Braun V \& Clarke V (2006) Qual Res Psychol 3, 77-101 